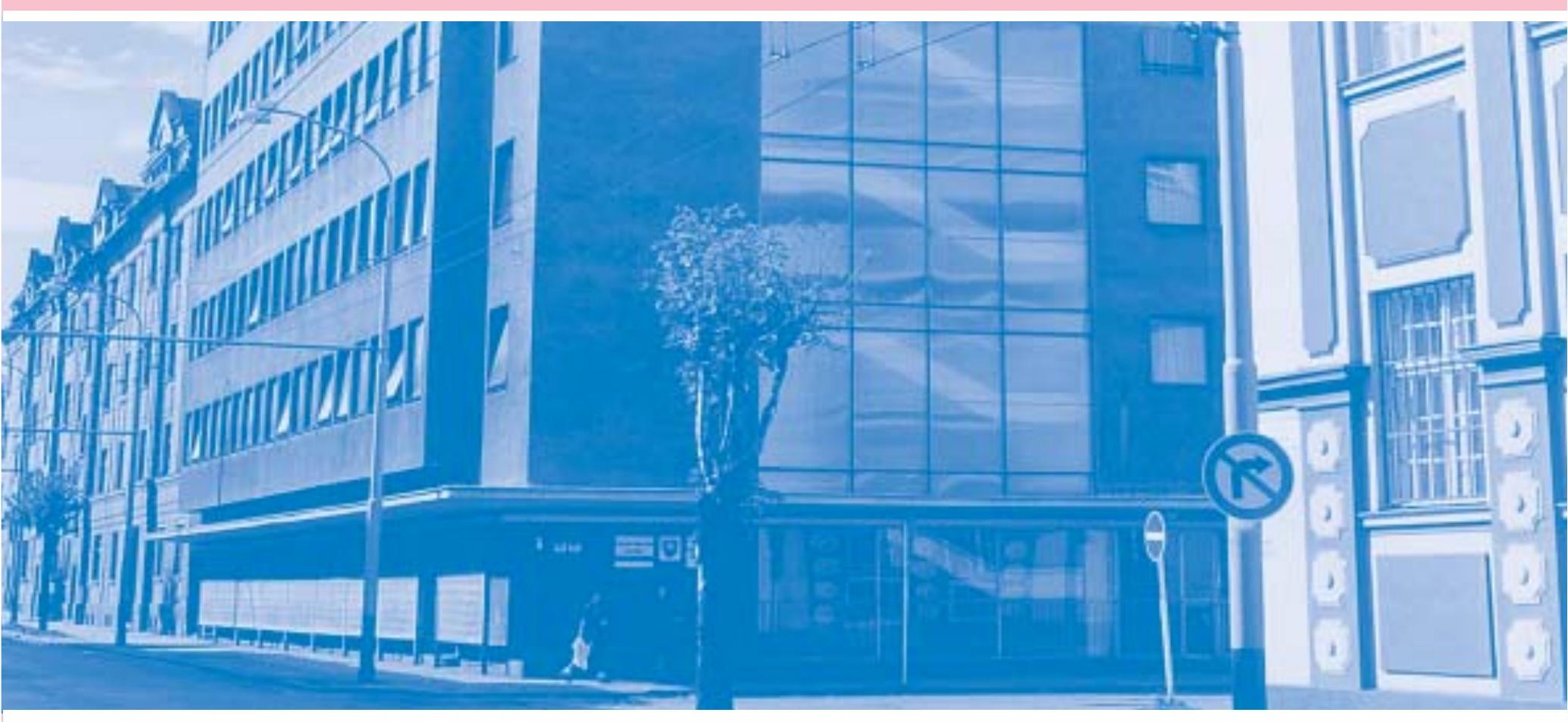

\title{
INSTITUTE OF INFORMATION AND COMMUNICATION TECHNOLOGIES
}




\section{INSTITUTE OF INFORMATION AND COMMUNICATION TEGHNOLOGIES}

\author{
Ústav informačných a komunikačných technológií \\ Moyzesova 20, 01026 Žilina, Slovak Republic \\ E-mail: skruvt@uvt.utc.sk \\ Phone: +421-41-7245704 \\ Fax: +421-41-5626961 \\ Director: Ing. Jozef Mužík
}

The roots of keeping computers and information technologies at the University of Žilina go back to 1967, whilst the Computer Centre was established as an independent part of the Department of Mathematics and Descriptive Geometry at the Faculty of Mechanical and Electrical Engineering. In 1982, the Computer Centre was transformed into the Institute of Computer Technology. In that time the institute serviced great mainframes where all computational needs of the university were solved and the first steps toward the university network had been done as well. The institute gradually extended its services as new technologies were emerging. In the year 2003 this all led to the new transformation and renaming of the institute into the Institute of Information and Communication Technologies. At present the institute provides support for all activities tied up with the information and communication technologies at the University of Žilina. Many services, the list of which follows, are provided:

- help desk for students and university staff;

- control center and maintenance of smart cards;

- design, installation and maintenance of the University's networks;

- maintenance of the computers and their HW and SW equipment,

- support of servers, system and application software for the Integrated University Information System;

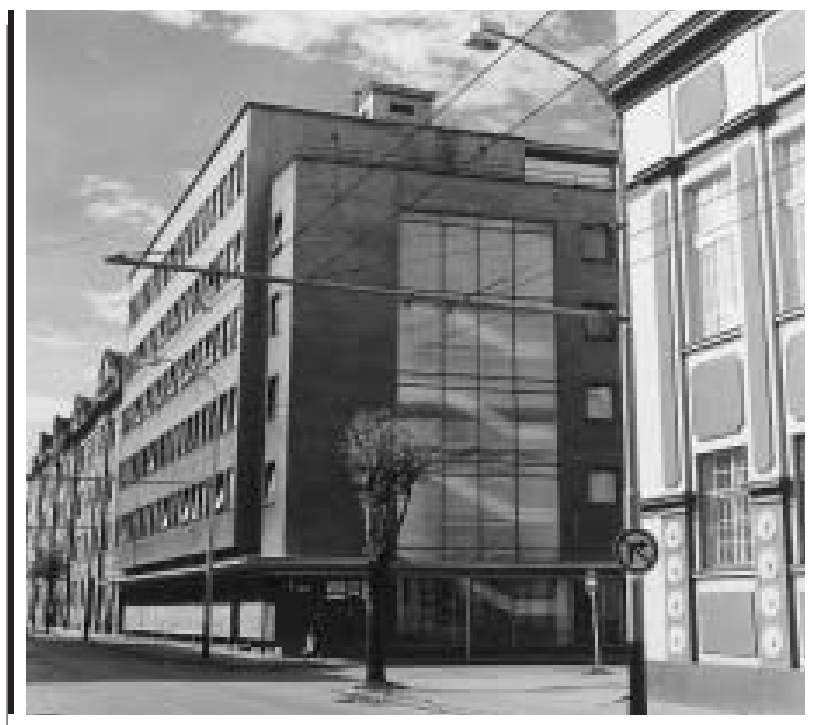

- consultancy to staff and students of exploitation of information and communication technologies in particular problems;

- negotiations of competitive software deals for the University;

- maintenance and expansion of the University's converged communication subsystem, (a combining of computer and telecom networks); - representation of the University at the Slovak Academic Network association (SANET) and at many other meetings as requested;

- support of the University's Management Information System;

- advises and help regarding computer-related multimedia issues to staff and students;

- maintenance of computer labs for open access and booked classes;

- support for e-learning

- keeping documentation on the major software programs in use at the University.

The Institute of Information and Communication Technologies consists of the following teams:

The Management Team

The Communication Technologies Team

The Computer Service Team

The Application Software Team

The Multimedia Technology Team 


\section{ACADEMIC METROPOLITAN NETWORKS AND SANET II}

\section{Introduction}

The origins of the recent campus computer network of the University of Žilina stem from February 1991, when the Institute of Computing Technology (ICT) became the member of the EARN and EUNET organisations. The first functional network connection was accomplished by means of PC and modem (MNP 5, 2400 bps) and in June 1991 by connection of the mainframe computer EC1045 to the Regional computing centre at the Czech Technical University (CVUT) in Prague. This led to establishment of the EARN node in Žilina.

ICT participated in the founding of the Slovak Academic Network organisation (SANET), which was established in April 1991. After the end of the operation of the EC1045 mainframe and retreat of the over ecstatic hype from the easy accessibility of the stand-alone PCs and terminal connections, ICT was chosen by the top management of the University in March 1992 to design and construct the University backbone network (backbone UTCNet, University of Transport and Communications Network). UTC-Net was conceived on the base of a multi-layer model, where the base layer forms the backbone. LANs of the Faculties form a higher level and the top layer forms the connection to SANET.

At the beginning, due to the lack of finance the circular topology was chosen. As the routers PCs $286 / 16 \mathrm{MHz}$ or PCs $386 \mathrm{SX} /$ $33 \mathrm{MHz}$ were used, equipped with KA9Q software and 19,2 kbps modems Gandalf LDS 720, used for short distance connections. In the new NF University building structured cabling was laid. Router and DEC server interconnected the backbone and digital telephone exchange Alcatel 5200. This enabled the use of the "data over voice" service for telephones with RS 232 interface. UTC-Net started its round 'o'clock operation on 10. 7. 1992.

UTC-Net developed since that time in many of its quantitative and qualitative attributes. They are the increase of speed and quality of individual connecting elements, change to star topology and mainly the change of ways and quality of connection with permanent fixed lines, radio lines and optical cables.

\section{SANET II}

The start of SANET was not an easy task, mostly because of economic reasons. A lot of effort was necessary to ascertain approval of the finance for the operation of SANET, for the membership fees for international networking organisations and for the international connection to the Internet networking structures from the ever-thin budget of the Ministry of Education. The Gov- ernment of the Slovak Republic by its decree No. 383/2001 decided to design and put into operation SANET II, the high-speed communication infrastructure with connection to the European communication network, connecting all the university centres in Slovakia. The Slovak government approved Decree no. 522/2001 on Policy of transfer to Information Society and joining of Slovakia to the of the eEurope + initiative.

After reaching the end of the I. Stage of the SANET II project in the first half of 2002, the following results have been reached:

1. Universities in Slovakia are connected to trans-European information highways.

2. The elementary goal of the network - to enable access to quicker Internet to as large number students as possible - is accomplished.

3. One part of the Slovak academic network has reached the same transfer speed as the speed of the networks in the most developed European countries - 1Gbit/s.

The elementary objective of the project - to help with the development of the Information Society in the Slovak Republic, by means of providing availability of the high speed networking for scientific, educational and research purposes, development of new applications, e.g. those enabling an easier access to information resources in EU and Slovakia, development of new services, as teleconferencing, distance education, medical consultation centres, etc.

Connection to the Trans-European network, developed by the Fifth and considered of utmost importance by the recent Sixth Framework Programmes will be made possible by the development of the high-speed network infrastructure in Slovakia. This will provide possibility of better co-operation with scientific and academic institutions in EU and easier inclusion of Slovak institutions in the international research and development projects. This should provide for:

1. Access to numerous information resources (databases) developed and maintained by Slovak institutions, or international information resources kept in Slovakia.

2. Provision of multimedia communication channel in the framework of educational services provided by Universities and as support for national and international scientific conferences for information interchanges.

3. Distance education (e-learning), which in future will provide the possibility to increase throughput of Slovak education in all of its various forms and levels.

4. Development of medical consultation centres, for provision of qualified telemedical services.

5. Provision of access to supercomputing capacities in the specialised European supercomputer centres. 
Among participating institutions in the SANET II project we can find universities, institutes of Slovak Academy of Sciences, research institutions, university hospitals and clinics. The access to SANET network is possible in 16 towns altogether in 21 nodes. The ultimate goal of the SANET II project is an optical backbone, connecting all university, educational and cultural centres, as: Bratislava - Trnava - Pieštany - Trenčín - Púchov - Žilina - Martin - Liptovský Mikuláš - Poprad - Tatranská Lomnica - Prešov Bardejov - Michalovce - Košice - Rožňava - Rimavská Sobota Lučenec - Banská Bystrica - Zvolen - Prievidza - Nové Zámky - Galanta - Dunajská Streda - Bratislava, by hired optical cables. The status of the SANET II network in January 2003 is shown in Fig. 1.

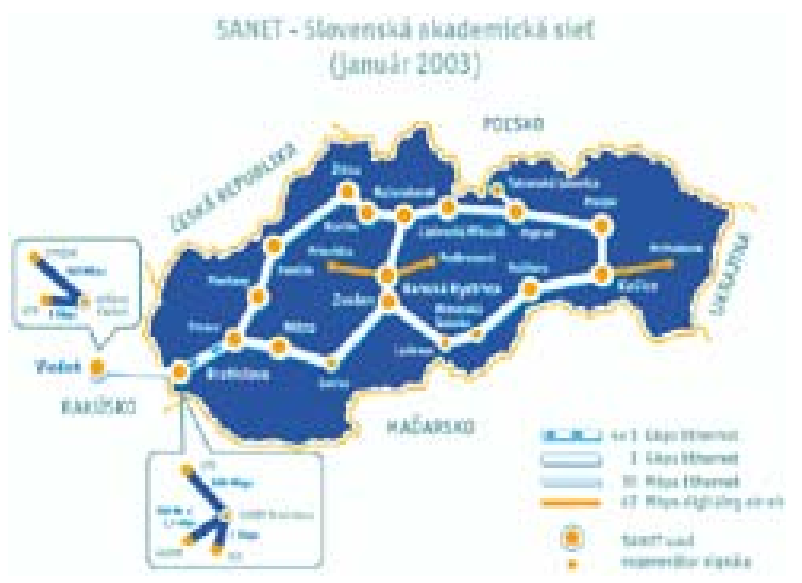

Fig. 1 SANET II January 2003

SANET as an institution is member of all important European networking organisations and since its conception participates in all projects of academic network infrastructure development in the EU framework, such as COSINE, ESSAT, TELESERV, EUROPANET, INSIDE. Thanks to participation in these projects and co financing from the European sources it was in the past possible to finance the development of the SANET network. The project supposes to connect the high-speed infrastructure to trans-European network through the participation in the European consortium GEANT (Gigabit European Advanced Network Technology). The central node of the SANET network (at CVT STU in Bratislava) serves as a node of the GEANT network in Slovakia as well. The agreement concerning connection with speed of $155 \mathrm{Mbps}$, with possible increase to $2.5 \mathrm{Gbps}$ has been signed already. To ascertain this international connectivity, $50 \%$ of the costs are covered by grant from EU. Further connection to the global network will be made through transatlantic operator EBONE. Further development should lead to interconnection with academic networks of the neighbouring countries (Czech Republic, Austria, Hungary).

The big advantage of the SANET II project is the possibility of establishment of metropolitan networks in the University centres. The centres concerned are located in Bratislava, Košice, B. Bystrica, Nitra, Žilina and Trnava. It is supposed that the transfer speed in the metropolitan networks should be $100 \mathrm{Mbps}$ or $1 \mathrm{Gbps}$. It is suggested that such infrastructure can be developed with proprietary or hired cables with the Ethernet protocol use.

\section{Academic metropolitan network of the University of Žilina}

At the same time as the project SANET II started, the development of the Academic metropolitan network of the University of Žilina started as well. The development consisted of preparation and realisation of the university metropolitan network, transformation of the networks of faculties, based on thin and thick Ethernet technologies and definition of the base parameters of the university metropolitan network. New infrastructure should connect all-important localities of the University. The technology used should be financially accessible, reliable and should enable future upgrades of the network with minimum costs.

The first stage of the realisation of the metropolitan network started in the beginning of the 2002 and was concluded in November 2002, at the same time as connection to SANET II. The Metropolitan network connects Vel'ký Diel campus - Faculty of Management Science and Informatics - administration building - Faculty of Civil Engineering - Student dormitories - University library. In the Vel'ký Diel campus area the following buildings areconnected to the network - the buildings NR, NS, new acquired building, technical building, Unimo H. During the first stage $7710 \mathrm{~m}$ of optical cable was laid.

The Metropolitan network is based on the switched Ethernet technology. A physical layer is built with optical threads (single mode, multimode) as structured metallic cabling of Category 5. Switched Ethernet provides possibilities for required quality of service and granted bandwidth of $10 / 100 \mathrm{Mbps}$ for the end user. The backbone of the academic MAN in Žiline has $1 \mathrm{~Gb}$ bandwidth. Such a breadth of the band makes possible e.g. transfer of $4 \mathrm{~GB}$ of data in $40 \mathrm{~s}$, parallel transfer of about 100 videoconferences in real time, video on demand (in the cable TV quality), voice over IP - transfer of voice in a very high quality. The sites which are still connected by coaxial cable (shared $10 \mathrm{Mb}$ Ethernet) cannot use services or applications provided by the switched Ethernet technology to a full extent. The fulfilment of the 2nd stage of the metropolitan area network is scheduled for the year 2003 . The project of connecting the Faculty of Special Engineering with optical cable and the Faculty Science with radio line to the Faculty of Civil Engineering node has been sent to SANET for approval.

The base parameters for the Academic metropolitan network of the University of Zilina were defined as follows: guarantee of bandwidth of $10 / 100 \mathrm{Mb}$ for the end user, Quality of Services (QoS) support, virtual networks support, multicasting support, central management of network, clustering, IP telephony, Video on Demand. 


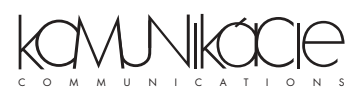

\section{Broadband networks}

\subsection{QoS (Quality of Service)}

Classical data networks, dedicated solely to the transfer of data, do not guarantee quality of the transport services. They developed independently of the voice services networks. Mostly the liberal environment, as compared with traditional telecommunication networks, usually monopolised by telecom operators, has fuelled rapid development of data networks. Continuous development and increase of available bandwidth and storage capacity in certain moment led to possibility of voice transfers over data networks. These technologies are either of the VoIP type (Voice over Internet Protocol), which "pack" digitised voice to IP protocol packets, or the VoFR type (Voice over Frame Relay), which "pack" digitised voice into the Frame Relay protocol frames. This possibility led to convergence of both types of networks. Recent data networks use the principle of switched packets. Transfers of voice and video however are very sensitive to transfer delays and jitter For these reasons guarantee of transfer services became necessary. The term QoS (Quality of Service) came into use. Support of QoS is based on the idea that transfers speeds, intensity of errors and further transfer parameters are measurable entities and therefore there is possibility of their improvement and provision of guarantee on certain level. QoS especially concerns continuous (streamed) transfers of video and multimedia content in the broadband networks. In the networks it is very difficult to guarantee the use of common protocols based on the "best effort" principle. One possibility is to assign different priority levels to different types of data. These priorities enable packets with higher priority to pass through the inner nodes more quickly, than packets with lower priority. IP protocols do support use of priorities; its implementation in practice is rather rare. New ways of implementation are being searched. One of the possibilities is the use of MPLS protocol (MultiProtocol Label Switching), which speeds up the network traffic and gives possibility of its easier management. MPLS sets a specific path for a given sequence of packets, identifies the level set for each packet, which saves time necessary for the router to evaluate the address of the following node, to which the packet should be sent. The "Multiprotocol" designation in MPLS shows, that it can work with IP protocol, s ATM (Asynchronous Transport Mode) protocol and Frame Relay networking protocol. From the OSI reference model (RM OSI) point of view, protocol MPLS makes possible that more packets are routed in the second layer (switching) than in the third layer (routing). Another protocol which makes the switching in the third level of the RM OSI possible is the COPS (Common Open Policy Service) protocol. Transfer delay and jitter can be dealt also by other means. It is possible to use several reservation methods, which use the principle of putting aside (reserving) part of the transfer capacity available for transfers with specific requirements and demands. Reserved transfer capacity can be used according to the principle of circuit's interconnection. With this kind of interconnection used also by ATM transfer technology, it is possible to guarantee required transfer parameters. Reservation of certain transfer capacity in the TCP/IP networks can be ascertained by the use of RTP protocols
(Real Time Protocol) and/or RSVP protocol (Resource Reservation Protocol).

\subsection{Virtual networks}

Data networks are used concurrently by a number of independent users. Their use of addresses from the common address space makes communication among them possible. At the same time the current throughput of the network is dependent of the concurrent requirements of the users. Some tasks and users require certain degree of intimacy while using the network and its services. It is possible with use of certain technologies to create "insulated" networks within network, thus giving the user the feeling of proprietary "private" network. This is a mere illusion, so these networks are designated as Virtual Private Networks (VPN). A part of the VPN illusion is the independent own address space and the possibility of direct communication only among the participants of the concrete VPN. Communication with other "external" network users is possible only through the defined (and guarded) gate. "Logical" illusion may be enhanced in practical application by "physical" illusion, granting its user certain bandwidths. The biggest advantage of VPN is the possibility to maintain security of data transfers. The mechanism which serve as illusion of private network providers may serve at the same times as information encoders, the information is then transferred in its encrypted form and decoded at the user side.

Utilisation of the data networks requires significant financial and human resources and know-how. As a good "saving" practice outsourcing may be used. It may take a form of one time activity, such as development of the data network, or continuous activity, such as operation of the network, user support etc. At the same time this can concern private data network, owned and used by a single user.

\subsection{IP telephony}

IP protocol transfer attributes are in no way ideal for the voice transmission. Despite this undisputable fact, this protocol is widely used for telephony over the data networks. It is mostly due to the fact that data networks are widely available and therefore this effort for their multiple use for voice services as well. Solutions based on the IP protocol are used not only for interconnection of the exchange nodes of the operators in the network backbones, but also for connection to the current end users. To ascertain coexistence between data transfers based on the IP protocol and "classical" telephony, their interoperability is necessary. This interoperability as well as telephony in the IP data networks is codified by standard H.323. This standard has been developed by the International Telecommunication Union (ITU) for multimedia communication in the LAN environment, based on packet transfer, regardless of the guarantee of Quality of Services (QoS). It tackles as compulsory part audio (voice) communication, in the mandatory part video and data communication. Standard defined for the 
main elements supporting transfer of information in the packet networks:

- Terminals: These are the terminal nodes, enabling bidirectional communication in real time. Typical examples are "data phones", but classical PC with the software packages such as Microsoft NetMeeting may be used.

- Gateways: These serve for interconnection with classical telephone networks, based on the principle of circuit connection. Standard H.323 concerns packet networks.

- Gatekeepers: This is an equivalent of telephone exchange for H.323 compatible networks.

- Multipoint Control Units (MCU): Ascertain communication in case of conference-like transfers (among multiple participants).

All terminals compatible with H.323 have to support point-topoint communication. Bidirectional communication between two terminals may be managed by administrator of the respective zone or by MCU. Information content of the conference may be sent to individual terminals using the point-to-point mode. Multicast type of transfers may be transformed to series of unicast type of transfers and sent to individual terminals without specific support on the side of the network topology. MCU may possibly function in reverse - they may serve as receptors of more than one unicast transfers of point to point type and produce one stream of multicast data, used for transfer of information from one source to multiple concurrent users.

\subsection{Video on demand (VoD)}

Competition for the user and (at the same time) customer is rapidly developing in the video service domain. TV stations broadcast their programmes 24 hours a day through the Internet, where their broadcasting servers are positioned as nodes in the backbone. Many of these servers function on the Microsoft platform (making reception with the help of the "free" MS Media Player possible). It is nowadays possible to choose from different transfer speeds available, which gives possibility to adjust the reception to the actual bandwidth available. The video is shown in styled browser window the user side. In reality the player is embedded into the WWW page, showing downloaded or streamed video. There are still some problems with the players embedded into styled screen, e.g. with visibility of control buttons, with synchronous playing of sound track and with some codecs.

\section{Conclusion}

The practical implementation of the SANET II project provided possibilities for establishment and further development of academic metropolitan networks based on optical technologies. Technical limits of optical connections lie in the fantasy domain. As far as the network services are concerned, the throughput available makes services as software distribution by network, access to music files, videoconferencing, video on demand (VoD), Internet telephony (VoIP), and such possible. Concrete content on demand, enabling personalisation of the services may be provided. The term content networking begins with realisation of SANET II based on the optical connection to have its concrete contours.

\section{References:}

[1] Horváth P., Druga P.: Information and communication infrastructure - important priority of the process of the move towards information society. In: Proceedings of the conference Infosem 2002, (in Slovak), Pieštany, 2002

[2] Horváth, P.: Project SANET II - realisation of high-speed academic data network for science, research and education. In: Proceedings of the international conference - Information and communication technologies for all, (in Slovak), Bratislava, 2002

[3] Zelem, J.: Computer network of VŠDS - UTC-Net, In.: Proceedings of the national conference Informatika '93, Information technologies and computing technology in educational institutions, pp. 197-200, (in Slovak), Bratislava, ÚIP ŠMT, 1993. 